\title{
Methotrexate in Psoriasis and Psoriatic Arthritis
}

\author{
Laura C. Coates (iD, Joseph F. Merola ${ }^{\mathbb{D}}$, Suzanne M. Grieb (D), Philip J. Mease (iD, \\ and Kristina Callis Duffin ${ }^{(1 D}$
}

\begin{abstract}
Methotrexate (MTX) is the most commonly prescribed first-line therapy in psoriatic arthritis (PsA) internationally and is also commonly used in the treatment of psoriasis. However, data supporting its use in PsA are limited and significant toxicities can occur. This article summarizes a debate at the 2019 Group for Research and Assessment of Psoriasis and Psoriatic Arthritis (GRAPPA) annual meeting that focused on the use of MTX in psoriasis and PsA. Four clinicians and 1 patient research partner presented clinical study data and the patient experience summarizing the efficacy, tolerability, and toxicity of MTX for both skin and musculoskeletal manifestations. A survey of attending GRAPPA members collected data on current and planned future use of MTX across the world. (J Rheumatol Suppl. 2020 June;96:31-5; doi:10.3899/jrheum.200124)
\end{abstract}

Key Indexing Terms:

PSORIASIS

GRAPPA
PSORIATIC ARTHRITIS

\section{PATIENT RESEARCH PARTNERS}

Despite limited evidence, methotrexate (MTX) is widely used internationally as an initial therapy in the treatment of psoriasis and psoriatic arthritis (PsA). In most countries, MTX is approved for use in psoriasis and rheumatoid arthritis (RA) but has not officially been approved for use in PsA. There are very few high-quality randomized controlled trials that support its use in the treatment of psoriasis or PsA. Therefore, its widespread use is presumably due to its assumed effectiveness (as shown in RA and psoriasis studies), its wide availability, and its low cost. In many

From the Department of Orthopaedics, Rheumatology, and Musculoskeletal Sciences, University of Oxford, Oxford, UK; Department of Dermatology and Medicine, Division of Rheumatology, Harvard Medical School, Brigham and Women's Hospital, Boston, Massachusetts; Department of Pediatrics, Research Associate, Johns Hopkins University School of Medicine, Baltimore, Maryland; Rheumatology Research, Swedish Medical Center/Providence St. Joseph Health and University of Washington School of Medicine, Seattle, Washington; Department of Dermatology, University of Utah, Salt Lake City, Utah, USA.

As part of the supplement series GRAPPA 2019, this report was reviewed internally and approved by the Guest Editors for integrity, accuracy, and consistency with scientific and ethical standards.

L.C. Coates is funded by a National Institute for Health Research (NIHR) Clinician Scientist award. The research was supported by the NIHR Oxford Biomedical Research Centre. The views expressed are those of the authors and not necessarily those of the UK National Health Service (NHS), the NIHR, or the Department of Health.

L.C. Coates, MB ChB, PhD, Department of Orthopaedics, Rheumatology, and Musculoskeletal Sciences, University of Oxford; J.F. Merola, MD, MMSc, Department of Dermatology and Medicine, Division of Rheumatology, Harvard Medical School, Brigham and Women's Hospital S.M. Grieb, PhD, MSPH, Department of Pediatrics, Research Associate, Johns Hopkins University School of Medicine; P.J. Mease, MD, Rheumatology Research, Swedish Medical Center/Providence St. Joseph Health and University of Washington School of Medicine; K. Callis Duffin, MD, MS, Associate Professor and Co-Chair, Department of Dermatology, University of Utah, and President, GRAPPA.

Address correspondence to L.C. Coates, Department of Orthopaedics, Rheumatology, and Musculoskeletal Sciences, University of Oxford, Botnar Research Centre, Windmill Road, Oxford, UK.

E-mail:laura.coates@ndorms.ox.ac.uk healthcare settings, MTX is required to be tried as a first-line therapy prior to access to other therapies with a strong evidence base such as biologics, thus enforcing its use.

At the 2019 Group for Research and Assessment of Psoriasis and PsA (GRAPPA) annual meeting in Paris, France, a debate was held on the use of MTX in treating psoriasis and PsA. Suzanne M. Grieb, a GRAPPA patient research partner (PRP), presented her personal experience with MTX from the patient perspective on behalf of all GRAPPA PRP. Two dermatologists, Kristina Callis Duffin and Joseph F. Merola, and 2 rheumatologists, Laura C. Coates and Philip J. Mease, presented the evidence for and against the use of MTX in these indications. An audience survey was also held to investigate how GRAPPA members use MTX.

Dr. Callis Duffin presented a historical introduction to MTX that included its first use in a case series in the 1950 $\mathrm{s}^{1}$, key trials in psoriasis and PsA that have been published over the decades, and its most recent data from 2019 (Figure 1). At high doses, the mechanism of MTX is by competitive inhibition of dihydrofolate reductase, which interrupts the normal metabolism of tetrahydrofolate. Tetrahydrofolate is required for thymidine in DNA synthesis. Thus, MTX can inhibit the synthesis of DNA, RNA, thymidylates, and proteins. However, the mechanism of effect of low-dose MTX, as used in psoriasis and PsA therapy, is much less clear. At low doses in psoriasis, it has been shown to suppress $\mathrm{T}$ cell activation and adhesion molecule expression, which may be mediated by adenosine, polyglutamated MTX, or both ${ }^{2,3,4}$.

\section{Survey: Current Use of MTX \\ Using an audience response system, a live survey of participants was held to establish current MTX use among the GRAPPA annual meeting participants. Over 100 audience members responded to each question (56\% rheumatologists, $13 \%$ derma- tologists, $15 \%$ industry members, $8 \%$ trainees, and $7 \%$ PRP).}

Personal non-commercial use only. The Journal of Rheumatology Copyright @ 2020 . All rights reserved. 
1948

Sidney Farber

uses aminopterin

for childhood

leukemia
1951

Positive case

series in RA,

psoriasis
1962

Aminopterin

modified to

methotrexate
1964

First PsA double

blind study
1972

FDA approval PsO

AAD guidelines

\section{4}

Failed trial in PsA (Wilkens)
1988

FDA approval for RA
2008

CHAMPION

(ada v. mtx v

pbo for Pso)

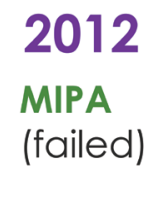

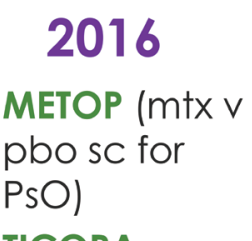

TICOPA
2019

SEAM (etn $v$

$\mathrm{m} t \mathrm{x}$

combo)

\section{(tight control)}

Figure 1. Key approvals and studies of MTX in psoriasis and PsA. RA: rheumatoid arthritis; MTX: methotrexate; PsA: psoriatic arthritis; FDA: US Food and Drug Administration; PsO: psoriasis; AAD: American Academy of Dermatology; ADA: adalimumab; MIPA: MTX in PsA; PBO: placebo; sc: subcutaneous; TICOPA: Tight Control of PsA; SEAM: Study of Etanercept And MTX; ETN: etanercept.

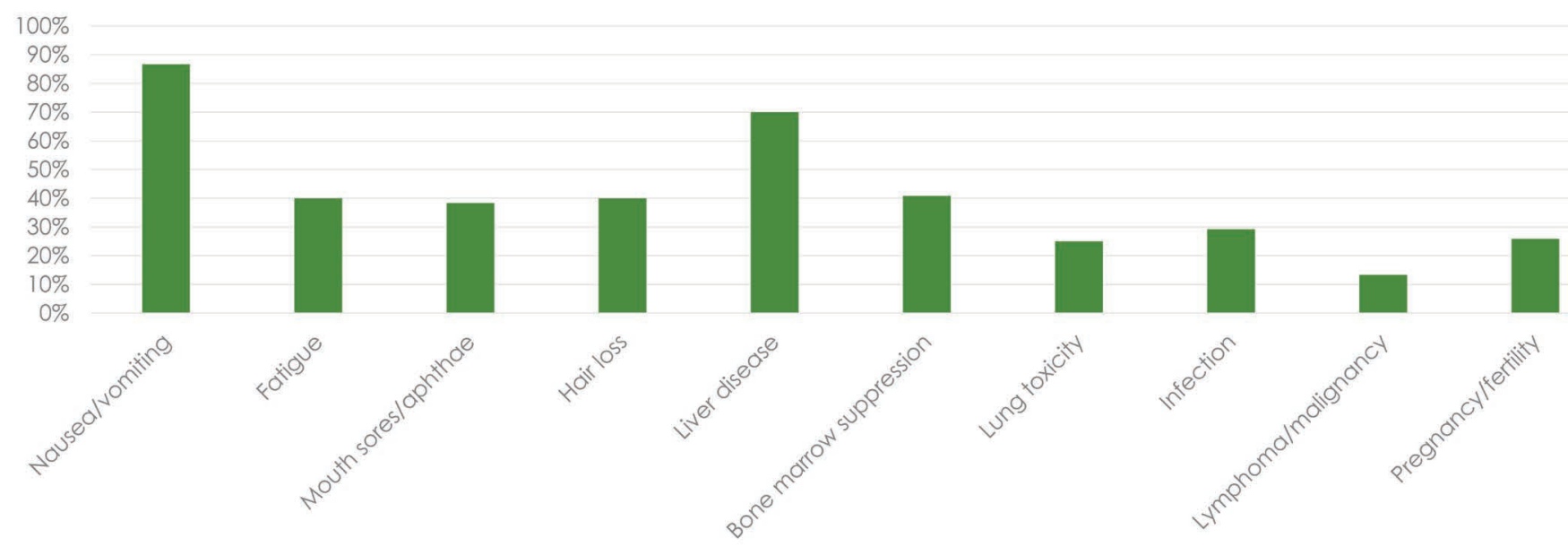

Figure 2. Audience survey results to the question, "In your initial discussion of MTX, which safety issues do you discuss?" MTX: methotrexate.

Of the audience members, $43 \%$ practiced in Europe, $33 \%$ in North America, $19 \%$ in South America, 5\% in Australasia, and $1 \%$ in Africa. MTX was most commonly prescribed once a week orally (74.6\%), subcutaneously $(16.7 \%)$, or intramuscularly $(1.8 \%)$. It was prescribed twice weekly orally by $4.4 \%$ and subcutaneously by $0.9 \%$. Only $1.8 \%$ prescribed 3 doses of MTX every $8 \mathrm{~h}$ once per week. The most common starting dose was $15 \mathrm{mg}$ for just over half the respondents, with a range from $5 \mathrm{mg}$ to $25 \mathrm{mg}$. The most common maintenance dose was $20 \mathrm{mg}$, but about $20 \%$ indicated their usual dose was either 15 $\mathrm{mg}$ or $25 \mathrm{mg}$. Most (70\%) said their maximum dose was 25 $\mathrm{mg}$, but some (16\%) limited their dose to $20 \mathrm{mg}$ or less. All physicians reported a co-prescription of folic acid, with most (50\%) giving a single weekly $5 \mathrm{mg}$ dose, and some (25\%) giving $1-2 \mathrm{mg}$ per day. The vast majority $(70 \%)$ monitored laboratory tests every 3 months for patients stable while taking MTX, with $27 \%$ checking bloodwork every 1-2 months. Physicians reported that MTX counseling included multiple safety and tolerability issues, with nausea and liver toxicity being most common (Figure 2).

When considering the use of MTX alongside biological disease-modifying antirheumatic drugs (bDMARD), the most common minimum dose to minimize immunogenicity was $10 \mathrm{mg}$, although some did not differentiate from their normal dosing. Practice for prescribing MTX alongside bDMARD was varied, with some (25\%) continuing MTX but tapering the dose, some (22\%) continuing MTX at the current dose, some (14\%) stopping MTX completely, and the largest group of respondents (35\%) using a variety of these approaches guided by individual circumstances and patient preference.

\section{Efficacy of MTX in Psoriasis and PsA}

To open the debate on MTX use, S.M. Grieb discussed per- 
sonal experiences from the GRAPPA PRP. Prior to the 2019 GRAPPA annual meeting, PRP were asked to respond to questions regarding their personal experience using MTX. Of the PRP, 11 of 13 responded, with 10 sharing their experiences, and 1 indicating no MTX use. Of those with experience using MTX, the majority used the tablet form, sometimes as monotherapy and sometimes in conjunction with additional medicines. Only 3 PRP had experience with the injectable form. Most of the PRP indicated that MTX did little to help their disease. The benefits they experienced were largely in the skin, while there was only a limited effect reported on arthritis. For the 3 PRP who responded well to the medication, the effect on their health and well-being was great. One PRP stated, "At first it was a revelation. Relatively quickly I was relieved of the feeling that I was moving my body through the weight of deep water, and movement became easier again, less painful. The psoriasis relief followed." However, for 2 of the 3 who found relief using MTX, it was unfortunately not sustained.

Dr. Merola introduced the data on MTX efficacy in psoriasis. He highlighted the CHAMPION study, a randomized trial comparing adalimumab (ADA), MTX, and placebo, which showed statistically significant efficacy compared to placebo for Psoriasis Area and Severity Index (PASI) 75\% improvement $(75)^{5}$. This study also showed ADA was clearly superior to MTX. He also presented data from the METOP study $^{6}$, a placebo-controlled randomized controlled trial of escalating doses of subcutaneous MTX. This study showed significant efficacy for MTX over placebo for PASI75 and PASI90, although rates for these outcomes were $51 \%$ and $24 \%$ at Week $24^{6}$.

Dr. Mease then provided a summary of the evidence for MTX use in PsA, including negative placebo-controlled, randomized controlled trials in $1984^{7}$ and $2012^{8}$. He acknowledged some positive data from open-label, uncontrolled studies such as the Tight Control of PsA (TICOPA) ${ }^{9,10}$ and RESPOND trials ${ }^{11}$ but was clear that, despite a lack of a specific psoriatic study, it was accepted that MTX was not effective in treating axial spondyloarthritis.

L.C. Coates then presented the data in more detail from the 2012 MTX in PsA (MIPA) study ${ }^{8}$. This study did not meet its primary endpoint (PsA Response Criteria) because there were a number of limitations in the study, including a relatively low target dose of $15 \mathrm{mg}$, a high rate of dropouts in both groups, and a slow recruitment process. Patients had much lower baseline disease activity than is usually seen in clinical trials, potentially limiting the sensitivity of the outcome measures used. Supplementary data from MIPA did suggest a more significant improvement in outcomes seen for those with polyarticular disease ${ }^{8}$. L.C. Coates also showed data from the TICOPA study, which suggest improvements in arthritis, enthesitis, dactylitis, and psoriasis in the first 12 weeks when patients received MTX only. At the end of the 48-week TICOPA study, around one-fourth of patients were in minimal disease activity (MDA) while taking MTX alone, which suggests that MTX can lead to significant disease control for some patients ${ }^{9}$.

Dr. Mease showed data from 2 recent head-to-head studies comparing MTX with tumor necrosis factor inhibitors (TNFi) in PsA. The RESPOND study, an open-label comparison of MTX and infliximab, showed clear superiority for infliximab but also a high response rate for those treated with MTX, with an American College of Rheumatology 20\% improvement (ACR20) of $66.7 \%$ at Week $16^{11}$. More recently, the Study of Etanercept And MTX (SEAM) compared MTX monotherapy with etanercept (ETN) monotherapy, as well as a combination of both drugs. Unlike the RESPOND study, this was double-blind. Again, this study confirmed the superiority of TNFi inhibitors over MTX in clinical, patient-reported, and radiographic measures. It also showed marked improvements in arthritis, psoriasis, enthesitis, and dactylitis in those receiving only $\mathrm{MTX}^{12}$. The primary endpoint, ACR20 at Week 24, was $60.9 \%$ for combination MTX/ETN versus $50.7 \%$ for MTX alone, while MDA (a key secondary endpoint) was met by $35.7 \%$ in the combination arm versus $22.9 \%$ receiving MTX monotherapy. Unlike studies in RA, in which the combination of a TNFi and MTX was shown to have superiority of effect over monotherapy with either drug, the SEAM study demonstrated that there was no superiority of the combination of ETN plus MTX versus ETN alone, except in skin measures ${ }^{12}$. This observation is reassuring for those clinicians and patients who prefer not to use the combination.

Dr. Merola presented a summary of additional data on the use of MTX. There is some evidence, although mostly in the fields of RA and Crohn disease, that MTX may be helpful in reducing the development of antidrug antibodies and boosting trough levels of MTX $^{13,14,15}$. He also presented data on the dosing and bioavailability of MTX from RA studies. The first pharmacokinetic (PK) study showed that the bioavailability of MTX given orally seems to plateau at 15-20 mg per week, but that MTX given subcutaneously showed no plateau up to a 25-mg weekly dose ${ }^{16}$. The second PK study showed that bioavailability was significantly higher (28\%) when MTX was given as a split dose $8 \mathrm{~h}$ apart rather than in a single dose ${ }^{17}$.

The presenters summarized the data on MTX efficacy. MTX has established efficacy in plaque psoriasis and promising open-label or uncontrolled data in PsA, but response rates are clearly lower than alternative newer agents. A "number needed to treat" analysis was presented, which demonstrated that over 43 patients would need to be treated to get 1 patient to PASI90 response using MTX, compared to around 2 to 3 patients to achieve 1 patient's PASI90 for the newer, targeted therapies ${ }^{18}$.

\section{Tolerability}

In the next section, S.M. Grieb started the discussion on

Personal non-commercial use only. The Journal of Rheumatology Copyright $(\subset) 2020$. All rights reserved. 
MTX's tolerability. Most of the PRP who had taken MTX had experienced side effects ranging from minor nausea, light-headedness, stomach cramping, and soreness at the injection site, to more severe symptoms such as vomiting and depression. Two PRP noted that MTX increased their fatigue. One PRP was unsure whether side effects occurred and commented, "I don't think that it had any really obvious side effects with me. Though with everything else going crazy, side effects could have been easily missed in the mix."

Dr. Mease then reported on adverse events in the double-blind SEAM study. There was a clear difference in reports of nausea with rates of $6 \%$ with ETN alone but $13-14 \%$ in the MTX arms $^{12}$. This was echoed in the METOP study of patients with psoriasis in which $14 \%$ reported nausea, even with injectable $\mathrm{MTX}^{6}$. Interestingly, rates of abdominal pain and diarrhea were shown to be similar to placebo or ETN groups in both of these studies ${ }^{6,12}$. L.C. Coates then summarized data on MTX durability in clinical practice, which is heavily influenced by issues of tolerability ${ }^{19,20}$. At the end of the TICOPA study, over $80 \%$ of patients were taking MTX, either alone or in combination, but at a recent followup (at 5 or more yrs since the end of the study), only around 50\% still took the drug. A study in Cambridge, UK, found about $60 \%$ of patients continued MTX for PsA, but the majority of those who stopped MTX did so because of tolerability concerns ${ }^{20}$.

\section{Toxicity}

S.M. Grieb again relayed the PRP experience, in which the majority had fortunately not experienced signs of toxicity. One PRP who responded well to MTX had to stop taking it owing to signs that it was causing serious physical issues. Another PRP noted liver tolerance problems, with alanine aminotransferase remaining high while taking the medicine. Although most PRP had not experienced significant toxicity, PRP still expressed significant concerns about this topic, primarily around counseling for potential longterm effects of MTX use while taking the drug, but also the potential effect after a patient discontinues treatment. A common concern that the PRP expressed was the lack of clarity between the use of MTX for PsA and its use for cancer treatment. One PRP raised a concern about toxicity while taking MTX as a woman of childbearing age. Regular questioning about potential pregnancy and her concerns about fetal toxicity made her question why she was allowing MTX into her body, particularly because it did not seem to be effective.

The panelists presented a brief review of safety and highlighted MTX's effects on bone marrow and the liver, as well as its potential for lung toxicity. A number of special populations were highlighted in which MTX would be contraindicated or used with additional caution, including those who have active malignancy, active infection, recurrent infection, hemochromatosis, a history of excess alcohol intake, plans for conception, or current pregnancy. Regular monitoring is always recommended with MTX use, to identify potential toxicity in the bone marrow or liver. An analysis in the Corrona registry highlighted the increased risk of elevated liver enzymes with MTX, with $1-2 \%$ of patients showing elevation above twice the upper limit of normal. Higher risks were seen in patients with PsA compared to patients with RA, as well as with higher MTX doses. The risk of MTX-associated lung toxicity may also be lower; no lung toxicity was identified in a metaanalysis of MTX use for non-RA inflammatory disease ${ }^{21}$.

\section{Survey: The Current and Future Role of MTX}

Following the presentations, a final audience survey was held to gather opinions on MTX's current and predicted future roles in clinical practice. Given the recent ACR recommendation to use TNFi first before oral small molecules for $\mathrm{PsA}^{22}$, the audience was asked, "If cost were no object, would you use TNFi first line for PsA?" A total of $69 \%$ answered yes, with rheumatologists and dermatologists responding similarly. There was variation in responses by geographical region, with first-line use of TNFi supported by $76 \%$ of Europeans, $88 \%$ of North Americans, but only $37 \%$ of South Americans.

The audience was then asked, "If cost were no object, which treatment would you use first line for moderate/severe plaque psoriasis?" Here, the majority voted for either interleukin (IL)-17 inhibitors (43\%) or IL-23 inhibitors (30\%), with $14 \%$ supporting the use of MTX. In this case, dermatologists and rheumatologists differed, with dermatologists choosing IL-17 and IL-23 inhibitors in identical proportions (30\%), and rheumatologists choosing IL-17 over IL-23 inhibitors (52\% vs $21 \%$ ), possibly because rheumatologists have a limited familiarity with IL-23 inhibitors. Again, there was some variation by geographical region; IL-23 inhibitors were the most popular North American choice (48\%), with IL-17 inhibitors as the most popular European (52\%) and South American (44\%) choice. MTX was chosen by $19 \%$ of South American participants, $12 \%$ of European participants, and only 5\% of North American participants.

Finally, because MTX has been acknowledged as the most commonly used first-line therapy for PsA worldwide, respondents were asked, "In 10 years' time, will MTX be the most commonly used drug for psoriatic disease in your region?" Here, the overall response was split with $51 \%$ answering yes and $49 \%$ answering no. Rheumatologists were more likely to say yes than dermatologists $(60 \%$ vs $36 \%$ ). While about 50\% of North American and European respondents answered yes, 68\% of South American participants thought MTX would still be the most commonly used first-line therapy.

\section{DISCUSSION}

The role of MTX in psoriasis and PsA remains controversial. While there is clear evidence of efficacy in psoriasis, the

Personal non-commercial use only. The Journal of Rheumatology Copyright $(\subset) 2020$. All rights reserved. 
data in arthritis are more limited. Recent head-to-head studies have offered some evidence to support MTX use in PsA, but these are uncontrolled data. Given the recent advances in therapies for both psoriasis and PsA in recent years, response rates for MTX look modest at best. MTX clearly raises significant concerns about tolerability and toxicity, although severe side effects are rare. GRAPPA members are split on MTX's future role, which was shown to be influenced by geographical location and access to therapies in different regions. Although efficacy is clearly inferior to newer, targeted therapies, MTX's low cost and widespread availability in many healthcare settings are important factors that contribute to its prominent role as a therapy in psoriasis and PsA.

\section{REFERENCES}

1. Gubner R, August S, Ginsberg V. Therapeutic suppression of tissue reactivity. II. Effect of aminopterin in rheumatoid arthritis and psoriasis. Am J Med Sci 1951;221:176-82.

2. Montesinos MC, Takedachi M, Thompson LF, Wilder TF, Fernandez P, Cronstein BN. The antiinflammatory mechanism of methotrexate depends on extracellular conversion of adenine nucleotides to adenosine by ecto-5'-nucleotidase: findings in a study of ecto-5'-nucleotidase gene-deficient mice. Arthritis Rheum 2007;56:1440-5.

3. Morabito L, Montesinos MC, Schreibman DM, Balter L, Thompson LF, Resta R, et al. Methotrexate and sulfasalazine promote adenosine release by a mechanism that requires ecto-5' -nucleotidase-mediated conversion of adenine nucleotides. J Clin Invest 1998;101:295-300.

4. Johnston A, Gudjonsson JE, Sigmundsdottir H, Ludviksson BR, Valdimarsson $\mathrm{H}$. The anti-inflammatory action of methotrexate is not mediated by lymphocyte apoptosis, but by the suppression of activation and adhesion molecules. Clin Immunol 2005;114:154-63.

5. Saurat JH, Stingl G, Dubertret L, Papp K, Langley RG, Ortonne JP, et al; CHAMPION Study Investigators. Efficacy and safety results from the randomized controlled comparative study of adalimumab vs. methotrexate vs. placebo in patients with psoriasis (CHAMPION). Br J Dermatol 2008;158:558-66.

6. Warren RB, Mrowietz U, von Kiedrowski R, Niesmann J, Wilsmann-Theis D, Ghoreschi K, et al. An intensified dosing schedule of subcutaneous methotrexate in patients with moderate to severe plaque-type psoriasis (METOP): a 52 week, multicentre, randomised, double-blind, placebo-controlled, phase 3 trial. Lancet 2017;389:528-37.

7. Willkens RF, Williams HJ, Ward JR, Egger MJ, Reading JC, Clements PJ, et al. Randomized, double-blind, placebo controlled trial of low-dose pulse methotrexate in psoriatic arthritis. Arthritis Rheum 1984;27:376-81.

8. Kingsley GH, Kowalczyk A, Taylor H, Ibrahim F, Packham JC, McHugh NJ, et al. A randomized placebo-controlled trial of methotrexate in psoriatic arthritis. Rheumatology 2012;51:1368-77.

9. Coates LC, Moverley AR, McParland L, Brown S, Navarro-Coy N, O'Dwyer JL, et al. Effect of tight control of inflammation in early psoriatic arthritis (TICOPA): A UK multicentre, open-label, randomised controlled trial. Lancet 2015;386:2489-98.
10. Coates LC, Helliwell PS. Methotrexate efficacy in the Tight Control in Psoriatic Arthritis study. J Rheumatol 2016;43:356-61.

11. Baranauskaite A, Raffayova H, Kungurov NV, Kubanova A, Venalis A, Helmle L, et al; RESPOND investigators. Infliximab plus methotrexate is superior to methotrexate alone in the treatment of psoriatic arthritis in methotrexate-naive patients: the RESPOND study. Ann Rheum Dis 2012;71:541-8.

12. Mease PJ, Gladman DD, Collier DH, Ritchlin CT, Helliwell PS, Liu L, et al. Etanercept and methotrexate as monotherapy or in combination for psoriatic arthritis: primary results from a randomized, controlled phase III trial. Arthritis Rheumatol 2019;71:1112-24.

13. Maini RN, Breedveld FC, Kalden JR, Smolen JS, Davis D, Macfarlane JD, et al. Therapeutic efficacy of multiple intravenous infusions of anti-tumor necrosis factor alpha monoclonal antibody combined with low-dose weekly methotrexate in rheumatoid arthritis. Arthritis Rheum 1998;41:1552-63.

14. Krieckaert CL, Nurmohamed MT, Wolbink GJ. Methotrexate reduces immunogenicity in adalimumab treated rheumatoid arthritis patients in a dose dependent manner. Ann Rheum Dis 2012;71:1914-5.

15. Keystone EC, Genovese MC, Klareskog L, Hsia EC, Hall ST, Miranda PC, et al; GO-FORWARD Study. Golimumab, a human antibody to tumour necrosis factor \{alpha\} given by monthly subcutaneous injections, in active rheumatoid arthritis despite methotrexate therapy: the GO-FORWARD study. Ann Rheum Dis 2009;68:789-96.

16. Schiff MH, Jaffe JS, Freundlich B. Head-to-head, randomised, crossover study of oral versus subcutaneous methotrexate in patients with rheumatoid arthritis: drug-exposure limitations of oral methotrexate at doses $\geq 15 \mathrm{mg}$ may be overcome with subcutaneous administration. Ann Rheum Dis 2014;73:1549-51.

17. Hoekstra M, Haagsma C, Neef C, Proost J, Knuif A, van de Laar M. Splitting high-dose oral methotrexate improves bioavailability: a pharmacokinetic study in patients with rheumatoid arthritis. J Rheumatol 2006;33:481-5.

18. Citrome L, Ketter TA. When does a difference make a difference? Interpretation of number needed to treat, number needed to harm, and likelihood to be helped or harmed. Int J Clin Pract 2013; 67:407-11.

19. Coates LC, Mahmood F, Freeston J, Emery P, Conaghan PG, Helliwell PS. Long-term follow-up of patients in the TIght COntrol of inflammation in early Psoriatic Arthritis (TICOPA) trial. Rheumatology 2019 Aug 31 (E-pub ahead of print).

20. Nikiphorou E, Negoescu A, Fitzpatrick JD, Goudie CT, Badcock A, Ostor AJ, et al. Indispensable or intolerable? Methotrexate in patients with rheumatoid and psoriatic arthritis: a retrospective review of discontinuation rates from a large UK cohort. Clin Rheumatol 2014;33:609-14.

21. Conway R, Low C, Coughlan RJ, O’Donnell MJ, Carey JJ. Methotrexate use and risk of lung disease in psoriasis, psoriatic arthritis, and inflammatory bowel disease: systematic literature review and meta-analysis of randomised controlled trials. BMJ 2015;350:h1269.

22. Singh JA, Guyatt G, Ogdie A, Gladman DD, Deal C, Deodhar A, et al. Special article: 2018 American College of Rheumatology/ National Psoriasis Foundation Guideline for the Treatment of Psoriatic Arthritis. Arthritis Rheumatol 2019;71:5-32. 